\begin{tabular}{l|l} 
REVISTA & $\begin{array}{l}\text { Revista Educación } \\
\text { ISSN: 0379-7082 } \\
\text { ISSN: 2215-2644 } \\
\text { revedu@gmail.com } \\
\text { Universidad de Costa Rica } \\
\text { Costa Rica }\end{array}$
\end{tabular}

\title{
Evaluación del trabajo en equipo en Educación Primaria. Estudio en España y México
}

Torres-Hernández, Norma; Gallego-Arrufat, María-Jesús

Evaluación del trabajo en equipo en Educación Primaria. Estudio en España y México

Revista Educación, vol. 46, núm. 1, 2022

Universidad de Costa Rica, Costa Rica

Disponible en: https://www.redalyc.org/articulo.oa?id=44068165020

DOI: https://doi.org/10.15517/revedu.v46i1.44258

\section{(c) (1) 90}

Esta obra está bajo una Licencia Creative Commons Atribución-NoComercial-SinDerivar 3.0 Internacional. 


\section{Evaluación del trabajo en equipo en Educación Primaria. Estudio en España y México}

Evaluation of the teamwork in Primary Education. An study in Spain and Mexico

Norma Torres-Hernández

Universidad de Granada, España

normath@ugr.es

iD https://orcid.org/0000-0003-4744-0313

Maria-Jesús Gallego-Arrufat

Universidad de Granada, España

mgallego@ugr.es

iD https://orcid.org/0000-0002-2296-5431
DOI: https://doi.org/10.15517/revedu.v46i1.44258 Redalyc: https://www.redalyc.org/articulo.oa? $\mathrm{id}=44068165020$

Recepción: 30 Octubre 2020

Aprobación: 10 Marzo 2021

\section{RESUMEN:}

El aprendizaje competencial ha tenido gran impacto en el ámbito educativo internacional. Las reformas educativas de España y México en educación básica en la última década plantean modelos en los que se destaca la evaluación de la habilidad de trabajo en equipo y el uso de rúbricas. Este artículo presenta un estudio cuasi-experimental, donde se ha usado la autoevaluación, la coevaluación y la evaluación docente para evaluar el trabajo en equipo $(\mathrm{N}=137)$. Para recoger información se utiliza una rúbrica diseñada ad hoc con ocho descriptores. El análisis de datos se lleva a cabo aplicando análisis exploratorios y pruebas estadísticas con ayuda del programa SPSS. Los resultados muestran que en la coevaluación cuatro descriptores son valorados con la nota máxima y seis en la autoevaluación, mientras que solo uno logra esta valoración por parte del profesorado. La media por grupos muestra que en la autoevaluación las puntuaciones son más altas. En los grupos experimentales existen diferencias significativas en la evaluación docente pero no en la evaluación entre pares. En los grupos control existen diferencias significativas solo en la autoevaluación. Cimentar valores del trabajo en equipo y su evaluación resulta fundamental en la educación primaria, y utilizar instrumentos como la rúbrica requiere formar en su elaboración y uso, tanto al alumnado como al profesorado.

Palabras Clave: Competencias para la vida, Enseñanza primaria, Evaluación del alumnado, Evaluación del profesorado, Trabajo en equipo.

\section{Abstract:}

Skills-based learning has had a significant impact on international educational community. Educational reforms of the basic education system in Spain and Mexico during the last decade, proposed educational models which required teamwork and student adherence to a rubric. A quasi-experimental study was conducted based on self-assessment, co-assessment, and teacher assessment to evaluate teamwork $(\mathrm{N}=137)$. Data was collected based on an ad hoc designed rubric with eight descriptors. Data analysis was done through exploratory analysis and statistical tests using the SPSS program. According to results of the co-evaluation, four descriptors received the maximum grade and six for the self-evaluation, while only one received this rating by the teaching staff. The average by groups shows that in the self-assessment the scores are higher. There were significant differences in the experimental group with regards to the teacher evaluation, but not in the peer assessment. The control groups also experienced significant differences, yet, only in the self-evaluation. Promoting the values of teamwork and assessment is fundamental in Primary Education and the use of instruments, such as the rubric, requires training both students and teachers for its preparation and use.

KEYWORDS: Life Skills, Primary Education, Student Evaluation, Teacher Evaluation, Team Work.

\section{INTRODUCCIÓN}

La incorporación del enfoque de aprendizaje por competencias en los marcos que regulan la educación, durante la última década, es uno de los aspectos con mayor impacto en el contexto internacional por sus 
implicaciones curriculares en el diseño, desarrollo y evaluación, también por su alta vinculación al contexto cercano y real del alumnado (Biesta, 2015; De la Orden, 2011).

Las reformas de los sistemas educativos proponen adaptar los currículos de los distintos niveles de enseñanza a las nuevas realidades de un mundo globalizado. Estas reformas han impactado a la enseñanza primaria, tanto en España como en México.

En España, las dos últimas leyes educativas son claro exponente de estas transformaciones que afectan al currículum básico: la Ley Orgánica para la Mejora de la Calidad Educativa (Jefatura de Estado, 2013) y la Ley Orgánica 2020 (LOMLOE) (Jefatura de Estado, 2020). En la actualidad, la evaluación se basa en objetivos y competencias establecidas con un carácter informativo, formativo y orientativo (Jefatura de Estado, 2020).

En México, el año 2019 marca una importante transformación en el ámbito educativo con la reforma constitucional al Artículo 3, el Acuerdo Educativo Nacional (AEN), la Nueva Escuela Mexicana y los cambios curriculares en los planes y programas de estudio para la educación básica y obligatoria. Estos cambios apuestan por la formación de personas capaces de adaptarse a mundos cambiantes y diversos, resolver problemas y trabajar colaborativamente (Secretaría de Educación Pública [SEP], 2019a). En cuanto a la evaluación, se insiste en la diversificación de instrumentos como las rúbricas (Secretaría de Gobernación [SG], 2019) y la combinación de heteroevaluaciones, coevaluaciones y autoevaluaciones (SEP, 2019a), esto en consideración de que el instrumento debe adaptarse al objeto evaluado.

Los marcos legislativos en ambos países coinciden en el valor de la evaluación con la participación del alumnado, la inclusión de las rúbricas como instrumento para evaluar y considerar el trabajo en equipo como una habilidad que forma parte de una competencia para la vida o para el aprendizaje permanente, según el Consejo de la Unión Europea, (2018).

Este estudio investiga sobre la evaluación del trabajo en equipo en estudiantes de sexto curso de educación primaria. Se usa una rúbrica ad hoc para que el profesorado evalúe el desempeño del alumnado y, a su vez, el alumnado evalúe a sus compañeros y compañeras durante el trabajo en equipo.

El propósito es describir las valoraciones del alumnado cuando se evalúa y evalúa a sus pares en una actividad realizada en equipo y las diferencias entre estas puntuaciones y las del profesorado cuando se usan o no las rúbricas.

\section{MARCo CONCEPTUAL}

El modelo educativo por competencias emerge como una tendencia innovadora promovida en todos los ámbitos de la educación (Coll, 2013; Díaz y Barroso, 2014). Según De la Orden (2011) su finalidad es preparar para el desempeño exitoso de funciones y para afrontar desafíos con una clara connotación de capacidad y aptitud en contextos de la vida real.

Las competencias son el «saber hacer» aplicado a una diversidad de contextos académicos, sociales y profesionales. Se ponen en acción conocimientos, habilidades y actitudes (Ministerio de Educación, Cultura y Deporte [MECD], 2014; Secretaría de Educación Pública [SEP], 2016b). Son transferibles en circunstancias particulares, en las que es posible e indispensable una comprensión del conocimiento y su vinculación con las habilidades prácticas o destrezas y actitudes que las integran (MECD, 2014). Por tanto, requieren expresarse de forma que el profesorado comprenda cómo debe apoyar al alumnado para desarrollarlas y sobre todo que puedan verificar, estudiante a estudiante, en qué medida las dominan.

Las competencias para la vida o para el aprendizaje permanente son aquellas combinaciones de conocimientos, habilidades, valores y actitudes que se requieren para afrontar exitosamente los desafíos diarios. Pueden expresarse y evaluarse en un continuo de grados a lo largo del proceso formativo en diversas etapas de enseñanza. Algunas de esas competencias se relacionan con habilidades sociales y actitudes necesarias en procesos de formación a lo largo de toda la vida. 
Su evaluación busca conocer cómo el alumnado organiza, estructura y usa sus aprendizajes en contextos determinados para resolver problemas de diversa complejidad e índole (SEP, 2016a). Este proceso valorativo se debe adaptar a las necesidades y características, suscitar aprendizajes y mejorar las competencias, tanto disciplinares como transversales. Se pretende hacer partícipe al alumnado en la valoración de sus logros y dificultades, con base en metas u objetivos claros cuando realizan determinadas tareas (Black y William, 1998).

Postulados del Acuerdo Educativo Nacional (SEP, 2019) y del Acuerdo 20/11/2019 (SG, 2019) de México, y de la LOMCE (Jefatura de Estado, 2013) y LOMLOE (Jefatura de Estado, 2020), en España, coinciden en señalar que la evaluación debe dejar de ser vista como mera calificación y certificación, para ser considerada como una ayuda a la adquisición o mejora de competencias. Estas tienen, como indicadores del éxito educativo, el desempeño eficiente de la función o rol para el que el alumnado se prepara a través de actividades. Exige una demostración práctica y evidencias del logro de la competencia.

De allí que, como una directriz clave de la evaluación, es importante valorar el desempeño individual y grupal del alumnado a través de tareas y proyectos colaborativos en los que se pone en juego las habilidades sociales, intelectuales, cognitivas, emocionales y humanas (García, 2011) para resolver y actuar ante diferentes problemas y situaciones sociales cercanas, significativas y funcionales para la vida como futura ciudadanía (Araujo et al., 2017) y que ayuden a mejorar el aprendizaje.

Tanto en México como en España se propone fortalecer el desarrollo de la capacidad de trabajo en equipo, que, según Camilloni (2010), aporta las siguientes ventajas: realización de tareas simultáneas y complejas que difícilmente se realizan individualmente, intervención de todas las personas del grupo, disminución de la ansiedad al participar en grupos pequeños, constancia de un ritmo de trabajo conjunto, mejoramiento del sentido de responsabilidad, estimulación de la formación de grupos de trabajo y ayuda al profesorado a seguir el proceso, enseñar y orientar a cada equipo.

Según Araujo et al. (2017), trabajar en equipo permite compartir ideas y conocimientos. Para Ronquillo et al. (2014) forman al alumnado para tomar decisiones y llegar a acuerdos. El trabajo en equipo se plantea como una habilidad para toda la vida, que se promueve y adquiere a lo largo de los diferentes niveles educativos. Sin embargo, a pesar de su importancia, pocas veces se mide y evalúa su efectividad (Viles et al., 2012).

Las prácticas de evaluación en las que el alumnado es un elemento activo se proponen en ambos contextos, así como el uso de escalas de valoración o rúbricas para la evaluación como instrumentos adecuados para evaluar en este modelo por competencias (De la Orden, 2011), que busca que el alumnado comprenda mejor los objetivos de aprendizaje, sus criterios y el desempeño deseado (Rigo, 2016).

Las rúbricas, también llamadas escalas de valoración, de acuerdo con Martínez-Rizo (2012), constituyen una estrategia para explorar posturas, habilidades, actitudes u opiniones del alumnado. Su uso promueve la evaluación formativa, pero también se suelen utilizar para la evaluación sumativa. En ambos casos se pretende la adquisición de competencias orientadas al aprendizaje desde la reflexión y valoración a partir del reconocimiento de sus propias dificultades y fortalezas, así como las de sus compañeras y compañeros en las actividades de tipo colaborativo. Todo lo anterior es bajo la coordinación del profesorado en su seguimiento, orientación y evaluación, donde sus actuaciones son fundamentales en la labor educativa (Ruiz, 2012). Se propone realizar prácticas valorativas con participación del alumnado mediante una autoevaluación y coevaluación (SEP, 2019a; Tur et al., 2019) que generen confianza y responsabilidad en la ejecución de las tareas (MECD, 2014).

La coevaluación puede favorecer el sentido de responsabilidad entre el alumnado y formar conciencia sobre las implicaciones de evaluar con honestidad. Los debates e interacciones que se producen durante la coevaluación se caracterizan por el uso de un 'lenguaje entre iguales' y por una elevada implicación con valoraciones que pueden ser tanto o más efectivas (Montanero et al., 2014) para mejorar la tarea que será evaluada. 
La rúbrica contiene descriptores cualitativos que establecen la naturaleza del desempeño, apoyada en perspectivas de criterio y no en referencia a una norma (Simon y Forgette, 2001), ya que orienta el análisis de procesos o productos de los esfuerzos del alumnado (Moskal, 2000) a partir de lo que se quiere evaluar; informa sobre los niveles de desempeño con criterios específicos del logro a alcanzar en competencias y resultados de aprendizaje definidos por el profesorado (Gatica-Lara y Uribarren-Berrueta, 2013).

Como instrumento permite al profesorado innovar en el aula y contribuir a la mejora de los aprendizajes y competencias del alumnado (Dunn y Mulvenon, 2009; Mertler, 2001; Smit et al., 2017; Velasco-Martínez y Tójar-Hurtado, 2018), así como informar las valoraciones de forma muy rápida (Araujo et al., 2017). De acuerdo con Shipman et al. (2012), son una guía justa y equitativa de puntuación que permite al alumnado participar en su proceso valorativo por medio de la autoevaluación y la coevaluación (Harris et al., 2014; Panadero et al., 2012; Tur et al., 2019).

La evaluación que realiza la persona docente debe ser una estrategia sistémica de evaluación de desempeño y aspectos inspirados en un contexto y edad determinada, (Darling-Hammond, 2012) comprensibles y operativizados (Panadero y Jonsson, 2013) para que tengan un efecto positivo en el aprendizaje y fomenten una enseñanza eficaz y autorregulada (Panadero y Broadbent, 2018).

Estudios sobre rúbricas de evaluación en educación primaria

El uso de las rúbricas se ha extendido a todos los niveles educativos. Una razón de esta expansión apunta hacia el interés y la creciente aceptación de la llamada evaluación auténtica, con un enfoque cualitativo en el que se usa la rúbrica para evaluar aprendizajes y competencias (Aguilar, 2016). En educación primaria, este fenómeno ha ido creciendo; gran parte de los estudios tratan sobre la evaluación de contenidos curriculares en Matemáticas y Ciencias .Kowiyah y Mulyawati, 2018; Cankoy y Özder 2017; Peterman et al., 2015. Otros se centran en lengua o español (Aguilar, 2016; Bazán-Ramírez et al., 2015; Lozada et al., 2016; Sundeen et al., 2017. Sotomayor et al., 2015). Algunos estudios hacen referencia a la coevaluación y autoevaluación (Atienza y Valencia, 2019; Montanero et al., 2014; Montanero y Madeira, 2019)

En estudios como Kowiyah y Mulyawati (2018) o Peterman et al. (2015) definen las rúbricas como instrumento y método para evaluar las expectativas de rendimiento. Cuitiño et al. (2019) aprecian una variación favorable de puntuaciones en los criterios de la rúbrica cuando se aplica en dos momentos. Sisquiarco et al. (2018) analizan el desempeño oral con una rúbrica que facilita la retroalimentación. Kutlu et al. (2010a) consideran que las rúbricas ayudan a tener mejores resultados en el aprendizaje, que dan validez y fiabilidad a la evaluación (Cankoy y Özder, 2017; Uzun et al., 2018; Reza y Lovorn, 2010), y permiten mejorar conocimientos. Montanero et al. (2014) concluyen que la coevaluación genera indirectamente procesos de autoevaluación adicionales, resultado de las aportaciones, sugerencias o comentarios de los compañeros y compañeras. Montanero y Madeira (2019) señalan que las rúbricas son un importante apoyo para el logro de tareas, al utilizar, además, procesos de coevaluación. Atienza y Valencia (2019) hablan de honestidad del alumnado al evaluarse cuando se desliga la evaluación a la calificación y a la participación del alumnado, no solo en el proceso de evaluación, sino incluso en el mismo diseño del instrumento.

Usar rúbricas para evaluar es una práctica que gana terreno en la práctica educativa y en la investigación. Ello convierte al tema de este trabajo en una cuestión relevante y pertinente para el campo científico y, en particular, para el ámbito educativo.

\section{Metodología}

Este estudio es parte de una investigación que se lleva a cabo con cuatro grupos naturales constituidos en los contextos educativos de España (Esp.) y México (Méx.), con grupos experimentales (GE_Méx. y GE_Esp.) y grupos de control (GC_Méx. y GC_Esp.). Su diseño es cuasi-experimental con grupos de control no equivalentes (Bisquerra, 2014; Bono (2012); Ato et al., 2013). La asignación de los grupos experimentales y de control no fue al azar, sino por conveniencia, donde intervino el juicio de las personas investigadoras 
(Hernández, 2012) y criterios de voluntariedad del profesorado participante con interés en usar la rúbrica. Los cuatro grupos permanecieron intactos y definidos según estaban conformados en los centros educativos de ambos países.

Son objetivos de este estudio: describir y comparar los resultados de los descriptores de una rúbrica en una evaluación de $360^{\circ}$ (evaluación de la persona docente, autoevaluación y coevaluación) (O1); analizar las diferencias de las puntuaciones medias de los resultados por grupo (O2); y analizar la existencia o no de diferencias significativas en la evaluación intra e intergrupal de la autoevaluación, coevaluación y evaluación del profesorado en ambos contextos (O3).

Participantes

Participan 137 estudiantes de sexto de primaria. Del grupo 84 (66\%) son de nacionalidad mexicana y 53 (36\%) española. Asimismo, dos docentes de educación primaria de cada país. La distribución en porcentajes de alumnado participante según grupos naturales (GE_Méx., GE_Esp., GC_Méx. y GC_Esp.) se muestra en la Figura 1.

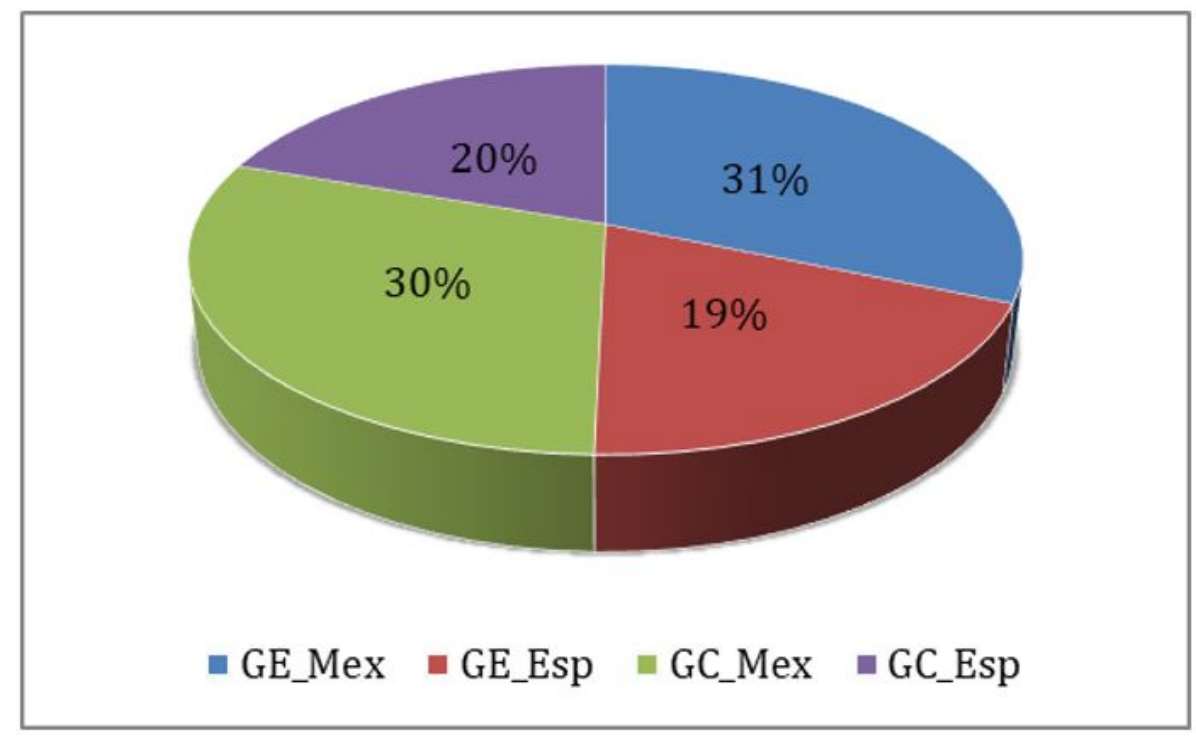

FIGURA 1.

Distribución del alumnado según grupos

Fuente: elaboración propia

En cuanto a la variable sexo, del total de los participantes 74 (54\%) son mujeres y 63 (46\%) son hombres. El profesorado participante son tres mujeres y un hombre con diferentes años de experiencia en la enseñanza primaria.

\section{Procedimiento}

Para la investigación se elaboró una rúbrica (Anexo I) con ocho descriptores distribuidos en las dimensiones aportaciones individuales (AI) y habilidades sociales (HS) (Tabla 1). 
TABLA 1

Dimensiones y descriptores de la rúbrica trabajo en equipo

\begin{tabular}{lll}
\hline Dimensiones & Descriptores & Código \\
\hline \multirow{3}{*}{ Aportaciones individuales (AI) } & Aportación de ideas y conocimientos útiles al trabajo del grupo & A \\
\cline { 2 - 3 } & Búsqueda de información en Internet y otras fuentes & RA \\
\cline { 2 - 3 } & Realización de las actividades asignadas & R \\
\cline { 2 - 3 } Habilidadesar a tiempo la tarea lactividad & RTE \\
\hline & Respeto a la opinión y los acuerdos tomados por grupo & RC \\
\cline { 2 - 3 } & Respeto al trabajo de las personas integrantes del equipo & CyCT \\
\cline { 2 - 3 } & Escuchar con atención las aportaciones de los compañeros y compañeras del grupo y de la clase & HSE \\
\cline { 2 - 3 } & Concentración y colaboración en el trabajo & CyCT \\
\hline
\end{tabular}

Fuente: elaboración propia

Fue creada ad hoc para este estudio y se validó en los dos contextos, donde se obtuvo: $\alpha=.956$ en España y $\alpha=.825$ en México. Su aplicación fue parte de una intervención educativa para la mejora de las competencias básicas en educación primaria. La Figura 2 muestra el proceso de elaboración, validación y aplicación.

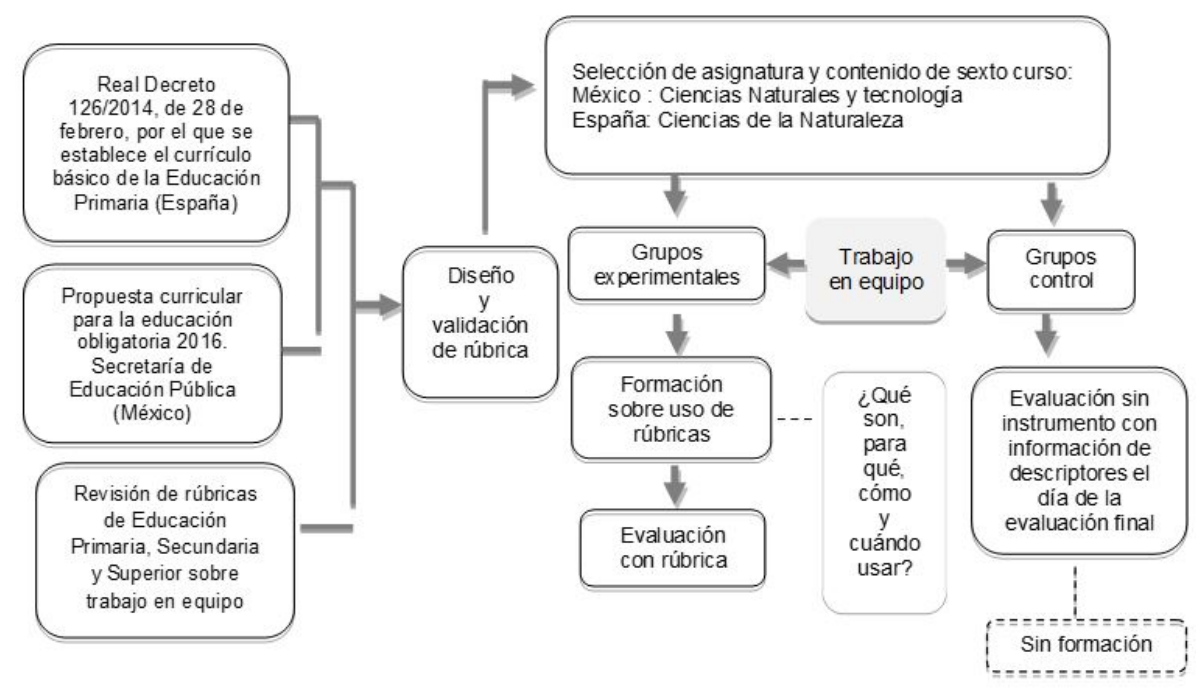

FIGURA 2

Proceso seguido en el estudio

Fuente: elaboración propia

Los datos obtenidos de la aplicación de la rúbrica son las puntuaciones otorgadas por alumnado y profesorado, tanto de México como de España. Con ellos se hizo el siguiente análisis: para el objetivo 1 se exploran los resultados de los ocho indicadores de la rúbrica, con especial atención a los resultados de estadística descriptiva para valoraciones de los tres tipos de evaluación de los cuatro grupos. Para el objetivo 2 se exploran resultados, con énfasis en la media obtenida por grupo en los tres tipos de evaluación; se comparan resultados entre grupos y entre contextos. Para el objetivo 3, comprobados los supuestos de normalidad de los datos, se aplicó la prueba no paramétrica U de Mann-Whitney para determinar si existen o no diferencias significativas entre los resultados de la autoevaluación, coevaluación y evaluación del profesorado. Se comparan resultados de grupos experimentales y control por país y entre países. 


\section{Resultados}

El análisis exploratorio de los ocho indicadores de la rúbrica muestra en la Figura 3 las medias por cada grupo y descriptor de la rúbrica a partir de las valoraciones del alumnado y profesorado. En el objetivo 1 se registran los siguientes resultados:

En coevaluación, el alumnado valora su competencia de trabajo en equipo con la máxima nota en los siguientes descriptores: entrega a tiempo de la tarea (R), respeto a las opiniones y acuerdos del grupo (RTE), respeto al trabajo individual de sus integrantes (RC) y concentración y colaboración en el trabajo (CYCT). En autoevaluación, en seis de los ocho descriptores el alumnado se ha puntuado con la máxima nota: realización de las actividades asignadas (RA), entrega a tiempo de la tarea $(\mathrm{R})$, respeto de las opiniones y acuerdos del grupo (RTE), respeto al trabajo individual de sus integrantes (RC), escuchar con atención las aportaciones de compañeras, compañeros y del grupo (HSE), y concentración y colaboración en el trabajo (CYCT). Por parte del profesorado, solo el descriptor respeto al trabajo individual de sus integrantes (RC) obtuvo la valoración más alta. El descriptor $\mathrm{RC}$ es donde las puntuaciones son más elevadas en la coevaluación, autoevaluación y evaluación de la persona docente.

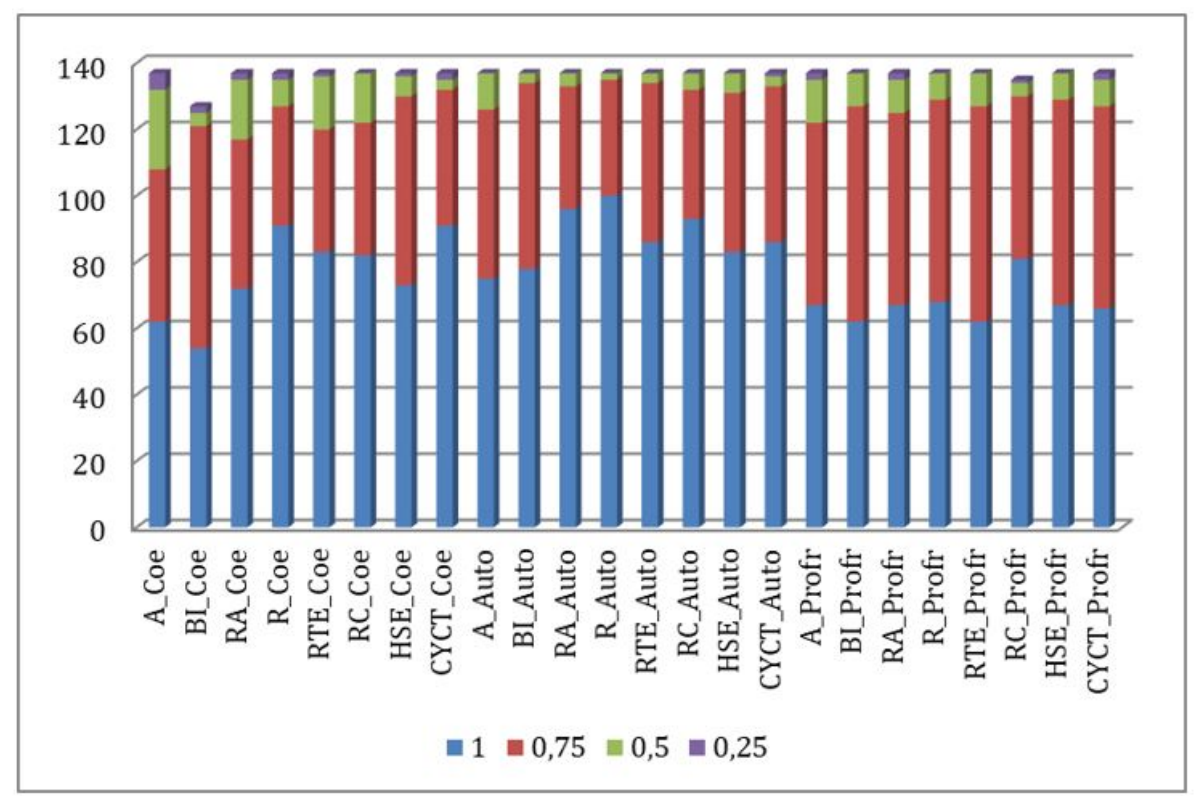

FIGURA 3.

Resultados comparativos por ítems al evaluar el trabajo en equipo (coevaluación, autoevaluación y evaluación de la persona docente)

Fuente: elaboración propia

La Tabla 2 muestra los resultados de las valoraciones agrupados en dos intervalos: $>0,75$ puntos y < 0,75 puntos. Los resultados representados en porcentajes muestran que, en la autoevaluación, en todos los descriptores de la rúbrica, el alumnado se evalúo por encima de 0,75 . 
TABLA 2

Porcentajes de valoraciones por descriptor de la rúbrica

\begin{tabular}{llllllllll}
\hline & \multirow{2}{*}{ Valoración } & \multirow{2}{*}{$\% \mathrm{~A}$} & $\%$ & $\%$ & \multirow{2}{*}{$\% \mathrm{k}$} & $\%$ & $\%$ & $\%$ & $\%$ \\
& & BI & RA & \% & RTE & RC & HSE & CYCT \\
\hline \multirow{2}{*}{ Coevaluación } & $>, 75$ & 79 & 88 & 85 & 93 & 88 & 89 & 95 & 96 \\
\hline \multirow{2}{*}{ Autoevaluación } & $<, 75$ & 21 & 2 & 15 & 7 & 12 & 11 & 5 & 4 \\
& $>, 75$ & 92 & 98 & 97 & 99 & 98 & 96 & 96 & 97 \\
\hline \multirow{2}{*}{$\begin{array}{l}\text { Evaluación del } \\
\text { profesorado }\end{array}$} & $<, 75$ & 8 & 2 & 3 & 1 & 2 & 4 & 4 & 3 \\
\hline
\end{tabular}

Fuente: elaboración propia

En el caso de las valoraciones de la persona docente, también las valoraciones están por encima de 0,75, excepto en el descriptor A, que en la coevaluación aparece con el porcentaje más bajo. En la coevaluación, en los descriptores BI, R, HSE y CYCT, el alumnado valoró mejor el desempeño de sus pares con $<0,75$.

Los resultados muestran que, en la coevaluación, en los descriptores BI, R, HSE y CYCT, hay valoraciones de más de 0,75 puntos. Igual sucede en la autoevaluación y la del profesorado cuando se valoran estos aspectos.

Con relación al objetivo 2, los resultados del análisis descriptivo, que comparan las valoraciones medias de cada uno de los cuatro grupos (Tabla 3), muestran que en el grupo experimental de México hay una valoración media mayor respecto al grupo experimental español. Entre los grupos de control, también las valoraciones totales del grupo mexicano superan a las valoraciones del grupo de España, si bien con una diferencia menor.

TABLA 3

Estadísticas descriptivas en los grupos experimentales y de control

\begin{tabular}{llllllll}
\hline Grupo & $\mathrm{n}$ & Rango & Min. & Máx. & Media & $\mathrm{s}$ & $\mathrm{s}^{2}$ \\
\hline GE_Méx & 43 & 0,19 & 0,79 & 0,98 & 0,8903 & 0,04851 & 0,002 \\
\hline GE_Esp & 26 & 0,40 & 0,58 & 0,98 & 0,8309 & 0,10706 & 0,011 \\
\hline GC_Méx & 41 & 0,23 & 0,75 & 0,98 & 0,8755 & 0,05966 & 0,004 \\
\hline GC_Esp & 27 & 0,27 & 0,72 & 0,99 & 0,8696 & 0,06021 & 0,004 \\
\hline
\end{tabular}

Fuente: elaboración propia

La Figura 4 muestra las medias obtenidas en coevaluación, autoevaluación y evaluación del profesorado en los cuatro grupos. Los resultados muestran mayor diferencia entre valoraciones de coevaluación y autoevaluación que entre la evaluación del profesorado y la autoevaluación, y entre la coevaluación en tres de los cuatro grupos: GE_Méx., GE_Esp. y GC_Méx. Por su parte, en el grupo GC_Esp., los resultados muestran mayor diferencia entre la autoevaluación y la evaluación del profesorado. 


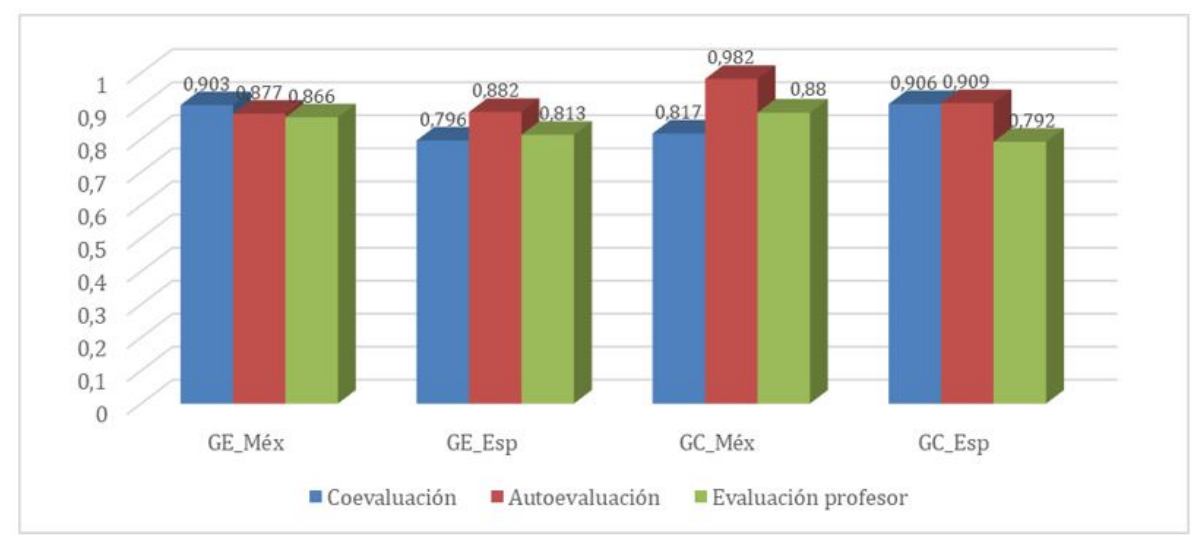

FIGURA 4.

Resultados comparativos de valores medios en los tres tipos de evaluación Fuente: elaboración propia

Al comparar grupos experimentales y grupos control entre países, los resultados muestran que, entre grupos experimentales, el grupo mexicano se valora más alto que el español en coevaluación. En autoevaluación, el español es más alto que el mexicano.

En los grupos de control, el español tiene valoraciones más altas en coevaluación que el mexicano. En autoevaluación, el mexicano más alto que el español.

Tanto en los grupos experimentales como en los grupos control mexicanos, la evaluación del profesorado es más alta que en el español.

Dado que el estudio se realiza con grupos independientes, en el objetivo 3 se analizó la posible existencia de diferencias entre las medias de los grupos experimentales y de control de ambos países y entre ambos países. En la Tabla 4 se muestran los resultados de la prueba no paramétrica U de Mann-Whitney, donde se resumen los datos de grupos experimentales y de control.

TABLA 4

Resultados estadísticos de prueba de los grupos experimentales y de control de ambos países

\begin{tabular}{lllllll}
\hline & México & \multicolumn{5}{c}{ España } \\
\hline Promedio & Coev. & Autoev. & Prof. & Coev. & Autoev. & Prof. \\
\hline $\begin{array}{l}\text { U de } \\
\text { Mann-Whitney }\end{array}$ & 472,500 & 591,000 & 848,000 & 201,000 & 299,000 & 320,500 \\
\hline W de Wilcoxon & 1333,500 & 1537,000 & 1709,000 & 552,000 & 650,000 & 698,500 \\
\hline$Z$ & $-3,684$ & $-2,631$ &,- 303 & $-2,685$ &,- 939 &,- 546 \\
\hline $\begin{array}{l}\text { Sig. asintótica } \\
\text { (bilateral) }\end{array}$ &, 000 &, 009 &, 762 &, 007 &, 348 &, 585 \\
\hline
\end{tabular}

Nota: Variable de agrupación: Tipo de grupo

Fuente: elaboración propia

Los resultados entre grupos de México muestran que en los rangos promedio de coevaluación $(\mathrm{p}=, 000)$ y autoevaluación $(\mathrm{p}=, 009)$ son menores al nivel de significancia $(, 05)$ y permiten indicar que existen diferencias significativas. En la evaluación del profesorado, el resultado de sus rangos $(\mathrm{p}=, 762)$ no muestra diferencias significativas. En el caso de los grupos de España, no hay diferencias significativas entre los rangos del 
promedio total en autoevaluación $(\mathrm{p}=, 348)$ y evaluación del profesorado $(\mathrm{p}=, 585)$, pero sí hay diferencias entre los rangos de promedio de la coevaluación $(\mathrm{p}=, 007)$.

Con la misma prueba se comparan los rangos promedio de los grupos experimentales (México-España) y de control (México-España). Los resultados muestran (Tabla 5) que, entre los grupos experimentales de ambos países, hay diferencias significativas en la autoevaluación del alumnado $(\mathrm{p}=, 910)$ y la evaluación del profesorado $(\mathrm{p}=, 044)$, pero no existen en la coevaluación $(\mathrm{p}=, 004)$ que realiza el alumnado en ambos países.

TABLA 5

Resultados de estadísticos de prueba de los grupos experimentales y control

\begin{tabular}{lllllll}
\hline & \multicolumn{2}{l}{$\begin{array}{l}\text { Grupos experimentales } \\
\text { (Méx_Esp) }\end{array}$} & & \multicolumn{3}{l}{ Grupos control (Méx_Esp) } \\
\hline Promedio & Coe. & Auto. & Profr. & Coe. & Auto. & Profr. \\
\hline U de Mann-Whitney & 331,000 & 550,000 & 397,5000 & 290,000 & 488,500 & 320,000 \\
\hline W de Wilcoxon & 682,000 & 1496,000 & 748,5000 & 1151,00 & 866,500 & 698,000 \\
\hline Z & $-2,842$ &,- 113 & $-2,012$ & $-3,318$ &,- 834 & $-2,942$ \\
\hline Sig. Sintótica (bilateral) &, 004 &, 910 &, 044 &, 001 &, 404 &, 003 \\
\hline Nota: Variable de agrupación: Tipo de grupo & & & & \\
\hline
\end{tabular}

Fuente: elaboración propia

En los grupos control de ambos contextos educativos hay diferencias significativas en la autoevaluación del alumnado $(\mathrm{p}=.404)$, pero no existe tal diferencia cuando este realiza su coevaluación $(\mathrm{p}=.001)$ o el profesorado evalúa el trabajo en equipo de sus estudiantes $(\mathrm{p}=.003)$.

\section{Conclusiones}

En España y México el trabajo en equipo es una habilidad de gran importancia en el futuro del alumnado de educación primaria.

Para esta investigación se elaboró, validó y utilizó una rúbrica diseñada con un propósito claro, para un colectivo concreto (Reza y Lovorn, 2010) y para diferentes contextos, por lo que se realizaron las adaptaciones correspondientes (Alsina et al., 2019; Fraile et al., 2017). Es una rúbrica comprensible para el alumnado y con un lenguaje sencillo.

En la coevaluación hay 4 descriptores en los que el estudiantado valora su competencia de trabajo en equipo con la máxima nota: R, RTE, RC y CYCT. En la autoevaluación, en 6 de los 8 descriptores, el alumnado se ha puntuado con la máxima nota: RA, R, RTE, RC, HSE y CYCT. Por parte del profesorado, solo en el descriptor RC la mitad del alumnado obtuvo la valoración más alta. De acuerdo a los mismos resultados, el descriptor RC es donde las puntuaciones son más elevadas en coevaluación, autoevaluación y en evaluación de la persona docente.

En cuanto al objetivo 1, con independencia del tipo de grupo y contexto, el alumnado valora más alto su desempeño en cuatro aspectos durante la coevaluación, seis en autoevaluación y en un descriptor de la rúbrica cuando lo evalúa el profesorado. En los cuatro grupos, el alumnado obtiene las puntuaciones más altas en la autoevaluación, por tanto, esto indica que el alumnado se otorga mayores puntuaciones cuando es esta misma población la que tiene la oportunidad de valorar su trabajo.

Para el objetivo 2 se comparan los tres tipos de evaluación y los resultados de los grupos experimentales y de control. Según el tipo de evaluación se concluye que las valoraciones más elevadas en la coevaluación aparecen en los grupos GE_Méx y GC_Esp. En la autoevaluación es el GE_Esp. La valoración media más 
baja en la evaluación de la persona docente se registra en el GE_Esp. Al comparar los tres tipos de evaluación se observa que las evaluaciones del profesorado tienden a ser, por lo general, más bajas cuando se comparan con la autoevaluación y la coevaluación.

Al comparar los grupos experimentales y grupos de control entre países, se concluye que hay valoraciones más altas en la coevaluación en GE_Méx., en autoevaluación en el GE_Esp. y en evaluación docente en México.

Luego de la aplicación de la prueba no paramétrica para el objetivo 3, se concluye que es la coevaluación donde se encuentran diferencias significativas en las comparaciones realizadas GE-GC_Méx. -GE-GC_Esp., GE_Méx-GE_Esp.y GC_Méx-GC_Esp.

Como conclusión general, se aprecia que, tanto en los ocho descriptores de la rúbrica como en los tres tipos de evaluación y en los cuatro grupos, hay puntuaciones altas. Esta evidencia difiere del estudio de Contreras et al. (2009), donde el uso de la rúbrica en educación primaria se aproximó hacia calificaciones bajas.

Si bien los resultados que aquí se presentan son de carácter cuantitativo, este estudio también originó información de tipo cualitativo, como el hecho de que el cuerpo docente de educación primaria no tiene suficiente información y conocimiento acerca de estas herramientas (Kutlu et al., 2010b; Knoch et al., 2007; Matshedisho, 2020). Cuando las usan, reconocen la necesidad de que, tanto docentes como alumnado, cuenten con una formación adicional sobre su uso.

Otro aspecto de interés es que valoran la evaluación de aspectos como los incluidos en la dimensión habilidades sociales, ya que influyen positivamente en la mejora de las actitudes. Este aspecto coincide en dos estudios (Kutlu et al., 2010a; Atienza y Valencia, 2019) en los que la evaluación con rúbricas de aspectos procedimentales y actitudinales es una de las futuras líneas de actuación por parte del profesorado y es transferible a otras asignaturas y etapas educativas.

En esta investigación se han encontrado bondades del uso de las rúbricas en los grupos experimentales, donde el alumnado se mostró animado y comprometido en el seguimiento del trabajo propio y de sus pares con una alta implicación (Montanero et al., 2014) y responsabilidad en el otorgamiento de notas a sus compañeras y compañeros.

Finalmente, al comparar los resultados de los grupos formados para el uso de la rúbrica y con conocimientos previos de los aspectos evaluables con aquellos que evaluaron su desempeño sin formación y el mismo día de la evaluación, se evidencia que las valoraciones en la coevaluación y autoevaluación fueron más cercanas a las personas docentes en los grupos experimentales, lo cual representa una ventaja para quienes conocían los criterios (Montanero y Madeira, 2019).

Esta investigación muestra el importante papel de las rúbricas de evaluación de competencias genéricas y transversales en alumnado de educación primaria. Evidencia su potencial para permitir la participación del alumnado en procesos de auto y coevaluación; es novedosa porque aporta una nueva mirada a la evaluación de las llamadas habilidades blandas, transversales o clave. Finalmente, confirma la utilidad de la rúbrica en la medida que hace menos distante la valoración entre alumnado y profesorado. Todo ello contribuye a mejorar procesos y prácticas de evaluación con una función más formativa.

La escasez de estudios que tratan sobre el trabajo en equipo en educación primaria y su evaluación, así como la dificultad para obtener información de tipo cualitativo sobre la experiencia por parte del alumnado, son algunas limitaciones de este estudio.

Por la importancia que se atribuye en México y España a la evaluación formativa, hay necesidad de continuar realizando investigaciones y estudios sobre el uso de las rúbricas en educación primaria para promover la participación del alumnado en la evaluación de competencias transversales con el mismo énfasis que las competencias disciplinares. Otra línea es realizar estudios longitudinales para conocer la evolución de los aspectos evaluables del trabajo en equipo a lo largo de la educación básica. Finalmente, estudiar las acciones formativas para la construcción de herramientas para evaluar de acuerdo a los modelos pedagógicos que vayan más allá de la mera sugerencia de uso de instrumentos por parte de las maestras y maestros. 


\section{REFERENCIAS}

Aguilar, P. (2016). Evaluación por rúbricas de la composición escrita en Educación Primaria. Anuari de Psicología, Monográfic: Educació, 79-111. https://doi.org/10.7203/17.2.79

Alsina, A., Ayllón, S. y Colomer, J. (2019). Validating the Narrative Reflection Assessment Rubric (NARRA) for reflective narratives in higher education. Assessment \& Evaluation in Higher Education, 44(1), 155-168. https: //doi.org/10.1080/02602938.2018.1486391

Araujo, D., Rochera, M.J. y Martínez, S.E. (2017). Evaluación de competencias en la primaria mexicana a través de un proyecto de Ciencias Naturales en la asignatura de Español. Revista Mexicana de Investigación Educativa, 22(73), 475-504. http://www.scielo.org.mx/scielo.php?

Atienza, R. y Valencia, A. (2019). Autoevaluación y evaluación compartida a través del uso de rúbricas. Una experiencia en educación primaria. Educación y Aprendizaje, 5(2), 40-47. https://doi.org/10.22370/ieya.2019.5.2.1499

Ato, M., López, J. y Benavente, A. (2013). Un sistema de clasificación de los diseños de investigación en psicología. Anales de Psicología, 29(3), 1038-1059. https://doi.org/10.6018/analesps.29.3.178511

Bazán-Ramírez, A., Barrera-Vázquez, D. y Vega-Alcántara, N. (2015). Validación de constructos de competencias de lectura y producción de textos en los inicio de la generalización de la reforma en la primaria Mexicana. Revista Iberoamericana sobre la Calidad, Eficacia y Cambio Educativo, 11(4), 61-76. https://revistas.uam.es/reice/artic le/view/2890

Biesta, G. (2015). What is education for? On good education, teacher judgement, and educational professionalism. European Journal of Education, 50(1), 75-87. https://doi.org/10.1111/ejed.12109

Bisquerra, R. (Coord.) (2014). Metodología de la Investigación Educativa. . (4ª Ed.). La Muralla.

Black, P. y Wiliam, D. (1998). Assessment and classroom learning. Assessment in Education, 5(1), 7-74. https://doi. org/10.1080/0969595980050102

Bono, R. (2012). Diseños cuasi-experimentales y longitudinales. Departamento de Metodología de Ciencias del Comportamiento. Universidad de Barcelona. http://hdl.handle.net/2445/30783

Camilloni, A. (2010). La evaluación de trabajos elaborados por grupo. En R. Anijovich, La evaluación significativa (pp.151-175). Paidós.

Cankoy, O. y Özder, H. (2017). Generalizability Theory Research on Developing a Scoring Rubric to Assess Primary School Students' Problem Posing Skills. EURASIA Journal of Mathematics Science and Technology Education, 13(6), 2423-2439. https://doi.org/10.12973/eurasia.2017.01233a

Coll, C. (2013). El currículo escolar en el marco de la nueva ecología del aprendizaje. Aula de Innovación Educativa, 219, 31-36. http://diposit.ub.edu/dspace/bitstream/2445/53975/1/627963.pdf

Contreras, L., González, M. y Urías, E. (2009). Evaluación de la escritura mediante rúbrica en la Educación Primaria en México. Revista Interamericana de Psicologia, 43(3), 518-531. http://pepsic.bvsalud.org/scielo.php?script=s ci_arttext\&pid=S0034-96902009000300011

Consejo de la Unión Europea. (4 de junio de 2018). Recomendación del Consejo de 22 de mayo de 2018 relativa a las competencias clave para el aprendizaje permanente. Diario Oficial de la Unión Europea. https://eur-lex.europa. eu/legal-content/ES/TXT/PDF/?uri=CELEX:32018H0604(01)\&from=SV

Cuitiño, J., Díaz, C. y Otarola, J. (2019). Promoción de la fluidez y precisión oral en inglés a través del role play. Cuadernos de Investigación Educativa, 10(1), 43-62. https://doi.org/10.18861/cied.2019.10.1.2880

Darling-Hammond, L. (2012). Desarrollo de un enfoque sistémico para evaluar la docencia y fomentar una enseñanza eficaz. Pensamiento educativo.Revista de Investigación Educacional latinoamericana 49(2), 1-20. http://www.p ensamientoeducativo.uc.cl/files/journals/2/articles/479/public/479-1357-1-PB.pdf

De la Orden, A. (2011). Reflexiones en torno a las competencias como objeto de evaluación en el ámbito educativo. Revista Electrónica de Investigación Educativa, 13(2). http://redie.uabc.mx/vol13no2/contenido-delaorden2.h tml 
Díaz, F. y Barroso, R. (2014). Diseño y validación de una propuesta de evaluación auténtica de competencias en un programa de formación de docentes de educación básica en México. Perspectiva Educacional, 53(1), 36-56. http s://www.redalyc.org/articulo.oa?id=3333/333329700004

Dunn, K. y Mulvenon, S. (2009). A Critical Review of Research on Formative Assessment: The Limited Scientific Evidence of the Impact of Formative Assessment in Education. Practical Assessment, Research y Evaluation, 4(14). https://doi.org/10.7275/jg4h-rb87

Fraile, J., Pardo, R. y Panadero, E. (2017). ¿Cómo emplear las rúbricas para implementar una verdadera evaluación formativa? Revista Complutense de Educación, 28(4), 1321-1334. https://doi.org/10.5209/RCED.51915

García, J. (2011). Modelo educativo basado en competencias: importancia y necesidad. Revista Electrónica Actualidades Investigativas en Educación, 11(3), 1-24. https://www.redalyc.org/articulo.oa?id=447/44722178014

Gatica-Lara, F. y Uribarren-Berrueta, T. (2013). ¿Cómo elaborar una rúbrica? Investigación en Educación Médica, 2(5), 61-65. https://doi.org/10.1016/S2007-5057(13)72684-X

Harris, L., Brown, G. y Harnett, J. (2014). Analysis of New Zealand primary and secondary student peer- and selfassessment comments: applying Hattie and Timperley's feedback model. Assessment in Education: Principles, Policy y Practice, 22(2), 265-281. https://doi.org/10.1080/0969594X.2014.976541

Hernández, O. (2012). Estadistica Elemental para Ciencias Sociales (3a ed.). Editorial Universidad de Costa Rica.

Jefatura del Estado. (2013). Ley Orgánica para la Mejora de la Calidad Educativa 8/2013, 9 de diciembre. Boletín Oficial del Estado, Núm. 295. https://www.boe.es/buscar/pdf/2013/BOE-A-2013-12886-consolidado.pdf

Jefatura del Estado. (2020). Ley Orgánica 3/2020, de 29 de diciembre, por la que se modifica la Ley Orgánica 2/2006, de 3 de mayo, de Educación. Boletín Oficial del Estado, Núm. 340. https://www.boe.es/boe/dias/2020/12/30/ pdfs/BOE-A-2020-17264.pdf

Knoch, U., Read, J. y von Randow, J. (2007). Re-training writing raters online: How does it compare with face-to-face training? Assessing Writing, 12(1), 26-43. https://doi.org/10.1016/j.asw.2007.04.001

Kutlu, O., Bilican, S. y Yildirim, O. (2010a). A study on the primary school teachers' attitudes towards rubrics with reference to different variables. Procedia Social and Behavioral Sciences, 2, 5398-5402. https://doi.org/10.1016 /j.sbspro.2010.03.880

Kutlu, O., Yildirim, O. y Bilican, S. (2010b). The comparison of the views of teachers with positive and negative attitudes towards rubrics. Procedia Social and Behavioral Sciences, 9, 1566-1573. https://doi.org/10.1016/j.sbs pro.2010.12.366

Kowiyah, K., y Mulyawati, I. (2018). An analysis of primary school students' representational ability in mathematics based on gender perspective. Journal of Physics: Conference Series, 948(1), 1-9. https://doi.org/10.1088/17426596/948/1/012016

Lozada, R., Martínez, L., Acle, G. y Ordaz, G. (2016). Validez y confiabilidad de una prueba para evaluar el desempeño de lectura y escritura en niños de primaria. En J. Castejón. Psicología y Educación. Presente y futuro. (pp.2239-2248). ACIPE.

Martínez-Rizo, F. (2012). Procedimientos para el estudio sobre las prácticas docentes. Revisión de Literatura. Revista Electrónica de Investigación y Evaluación Educativa 18(1), 1-22. https://ojs.uv.es/index.php/RELIEVE/article /view/2976/3688

Matshedisho, K. (2020). Straddling rows and columns: Students'(mis) conceptions of an assessment rubric. Assessment \& Evaluation in Higher Education, 45(2), 169-179. https://doi.org/10.1080/02602938.2019.1616671

Mertler, C. (2001). Designing scoring rubrics for your classroom. Practical Assessment, Research y Evaluation, 7(25). http://PAREonline.net/getvn.asp?v=7yn=25

Ministerio de Educación, Cultura y Deporte [MECD]. (2014). Real Decreto 126/2014, de 28 de febrero, por el que se establece el currículo básico de la Educación Primaria. Boletin Oficial del Estado, Núm. 52, 19349-19420. htt ps://www.boe.es/buscar/pdf/2014/BOE-A-2014-2222-consolidado.pdf

Moskal, B.M. (2000). Scoring rubrics: what, when and how? Practical Assessment, Research y Evaluation, 7(3). https: //northweststate.edu/wp-content/uploads/files/Moskal-ScoringRubrics.pdf 
Montanero, M., Lucero, M. y Fernández, M. (2014). Iterative co-evaluation with a rubric of narrative texts in Primary Education [Coevaluación iterativa con rúbrica de textos narrativos en la Educación Primaria, Infancia y Aprendizaje]. Journal for the Study of Education and Development, 37(1), 184-220. https://doi.org/10.1080/ 02103702.2014.881653

Montanero, M. y Madeira, M. (2019). Escritura colaborativa encadenada: efectos en la competencia narrativa de estudiantes de Educación Primaria. Journal for the Study of Education and Development, 42(4), 915-951. https ://doi.org/10.1080/02103702.2019.1650464

Panadero, E. y Broadbent, J. (2018). Developing evaluative judgement: A self-regulated learning perspective. En D. Boud, R. Ajjawi, P. Dawson y J. Tai (Eds.), Developing Evaluative Judgement: Assessment for Knowing and Producing Quality Work, (pp. 81-89). Routledge.

Panadero, E. y Jonsson, A. (2013). The use of scoring rubrics for formative assessment purposes revisited: A review. Educational Research Review, 9(0), 129-144. https://doi.org/10.1016/j.edurev.2013.01.002

Panadero, E., Tapia, J. A. y Huertas, J. A. (2012). Rubrics and self-assessment scripts effects on self-regulation, learning and self-efficacy in secondary education. Learning and Individual Differences, 6(22), 806-813. https://doi.org/ 10.1016/j.lindif.2012.04.007

Peterman, K., Cranston, A., Pryor, M. y Kermish, R. (2015). Measuring Primary Students' Graph Interpretation Skills Via a Performance Assessment: A case study in instrument development. International Journal of Science Education, 37(17), 2787-2808. https://doi.org/10.1080/09500693.2015.1105399

Rigo, D. (2016). Autorregulación y rúbricas como herramienta de evaluación. Experiencia desarrollada en educación primaria. Escuela Abierta, 19, 65-79. http://hdl.handle.net/11162/160586

Reza, A. y Lovorn, M. (2010). Reliability and validity of rubrics for assessment through writing. Assessing Writing, 15, 18-39. https://doi.org/10.1016/j.asw.2010.01.003

Ruiz, G. (2012). La Reforma Integral de la Educación Básica en México (RIEB) en la educación primaria: desafíos para la formación docente. Revista Electrónica Interuniversitaria de Formación del Profesorado, 15(1), 51-60. https:/ /www.redalyc.org/articulo.oa?id=2170/217024398004

Ronquillo, N., Gómez, M. y García, N. (2014). El uso de recursos multimedia para coadyuvar a la educación en valores en alumnas de un grupo de tercero de primaria. Didáctica, Innovación y Multimedia, 30. https://ddd.uab.cat/p ub/dim/dim_a2014m12n30/dim_a2014m12n30a5.pdf

Secretaría de Educación Pública [SEP]. (2016a). El modelo educativo 2016. El planteamiento pedagógico de la Reforma Educativa.SEP. https://www.gob.mx/cms/uploads/attachment/file/114501/Modelo_Educativo_2016.pdf

Secretaría de Educación Pública [SEP]. (2016b). Propuesta curricular para la educación obligatoria 2016. SEP. https:/ /www.gob.mx/cms/uploads/docs/Propuesta-Curricular-baja.pdf

Secretaría de Educación Pública [SEP]. (2019). Acuerdo Educativo Nacional. Implementación Operativa. SEP. https://infosen.senado.gob.mx/sgsp/gaceta/64/2/2019-11-14-1/assets/documentos/ Estrategia_Educacion_Inclusiva.pdf

Secretaría de Gobernación [SG]. (8 de noviembre de 2019). Acuerdo 20/11/19 por el que se modifica el diverso número $12 / 10 / 17$ por el que se establece el plan y los programas de estudio para la educación básica: aprendizajes clave para la educación integral. Diario Oficial de la Federación. https://www.dof.gob.mx/nota_detalle.php?co digo $=5578281 \&$ fecha $=08 / 11 / 2019$

Sisquiarco, A., Sánchez., S. y Abad, J. (2018). La influencia de la retroalimentación basada en estrategias en el desempeño oral de los estudiantes. HOW, 25(1), 93-113. https://howjournalcolombia.org/index.php/how/ar ticle/view/402/461

Smit, R., Bachmann, P., Blum, V., Brirri, T. y Hess, H. (2017). Effects of a rubric for mathematical reasoning on teaching and learning in primary school. Instructional Science, 45(5), 603-622. https://doi.org/10.1007/s1125 1-017-9416-2

Shipman, D., Roa, M., Hooten, J. y Zhan, J.W. (2012). Using the analytic rubric as an evaluation tool in nursing education: the positive and the negative. Nurse Education Today, 32(3), 246-249. https://doi.org/10.1016/j.n edt.2011.04.007 
Norma Torres-Hernández, et al. Evaluación del trabajo en equipo en Educación Primaria. Estudio en ...

Simon, M. y Forgette, R. (2001). A rubric for scoring postsecondary academic skills. Practical Assessment, Research y Evaluation, 7(18). http://PAREonline.net/getvn.asp?v=7yn=18

Sotomayor, C., Ávila, N. y Jéldrez, E. (Coord.) (2015). Rúbricas y otras herramientas para desarrollar la escritura en el aula. Grafhika

Sundeen, T., O’Neil, K. y Fanselow, S. (2017). Interpreting Visual Texts: Use of an Analytic Rubric of Evaluate Student Responses to Writing Prompts. Journal of Research in Childhood Education, 31(1) 9-27. https://doi.org/10.10 80/02568543.2016.1243174

Tur, G., Urbina, S. y Forteza, D. (2019). Rubric-based Formative Assessment in Process Eportfolio: Towards SelfRegulated Selfregulated Learning. Digital Education Review, 35, 18-35. https://revistes.ub.edu/index.php/der /article/view/22614

Uzun, B., Alici, D. y Aktas, M. (2018). Reliability of the Analytic Rubric and Checklist for the Assessment of Story Writing Skills: G and Decision Study in Generalizability Theory. European Journal of Education Research, 8(1), 169-180. https://doi.org/10.12973/eu-jer.8.1.169

Viles, E., Jaca, C., Campos, J., Serrano, N. y Santos, J. (2012). Evaluación de la competencia de trabajo en equipo en los grados de ingeniería. En 4th International Conference on Industrial Engineering and Industrial Management XIV Congreso de Ingeniería de Organización. 8-10 de septiembre. Donostia, San Sebastián. http://adingor.es/congresos/web/uploads/cio/cio2010/INNOVATION_IN_EDUCATION _AND_PROFESSIONALS_SKILLS//734-743.pdf

Velasco-Martínez, L. y Tójar-Hurtado, J. (2018). Competency-Based Evaluation in Higher Education-Design and Use of Competence Rubrics by University Educators. International Education Studies, 11(2), 118-132. https:/ /doi.org/10.5539/IES.V11N2P118

\section{Anexos}

Anexo 1

\begin{tabular}{|c|c|c|c|c|c|c|c|c|c|c|c|c|}
\hline Nombre de mi compañero o compañera & & tud & ian & & & udi & ant & & & tud & ian & \\
\hline $\begin{array}{l}\text { Aspectos que debo valorar de mis compañeros } \\
\text { o compañeras }\end{array}$ & 4 & 3 & 2 & 1 & 4 & 3 & 2 & 1 & 4 & 3 & 2 & 1 \\
\hline $\begin{array}{l}\text { Aporta ideas y conocimientos útiles al trabajo } \\
\text { del grupo. }\end{array}$ & & & & & & & & & & & & \\
\hline $\begin{array}{l}\text { Realiza las actividades que se le asignaron o a } \\
\text { las que se comprometió. }\end{array}$ & & & & & & & & & & & & \\
\hline $\begin{array}{l}\text { Entrega a tiempo la tarea /actividad por lo que } \\
\text { nadie del equipo tuvo que hacer trabajo extra. }\end{array}$ & & & & & & & & & & & & \\
\hline $\begin{array}{l}\text { Respeta la opinión de la mayoría y los } \\
\text { acuerdos que se toman en el interior del grupo. }\end{array}$ & & & & & & & & & & & & \\
\hline $\begin{array}{l}\text { Respeta el trabajo de las personas que } \\
\text { integran el equipo, sin criticar o burlarse de las } \\
\text { opiniones o posibles errores cometidos por } \\
\text { nuestro compañero o compañera. }\end{array}$ & & & & & & & & & & & & \\
\hline $\begin{array}{l}\text { Escucha con atención las aportaciones de la } \\
\text { compañera o compañero de equipo y de la } \\
\text { clase. }\end{array}$ & & & & & & & & & & & & \\
\hline $\begin{array}{l}\text { Durante las reuniones del grupo se concentra } \\
\text { en trabajar y colabora de manera responsable } \\
\text { en las actividades realizadas. }\end{array}$ & & & & & & & & & & & & \\
\hline $\begin{array}{l}3=0,75 \text { puntos } \\
\text { Buen trabajo }\end{array}$ & cien & & & & & & & & ora & & & \\
\hline
\end{tabular}




\section{INFORMACIÓN ADICIONAL}

Cómo citar: Torres-Hernández, N. y Gallego-Arrufat, M.-J. (2022). Evaluación del trabajo en equipo en Educación Primaria. Estudio en España y México. Revista Educación, 46(1). http://doi.org/10.15517/rev edu.v46i1.44258

Financiamiento: Esta investigación está financiada por el Plan Propio de Investigación de la Universidad de Granada, España. 